\title{
Optimized System for Extreme Precipitation Events Extraction Based on Improved Percentile Method
}

\author{
Chi Zhang ${ }^{1,2, *}$, Pu-wen Lei ${ }^{1}$, Koji Koyamada ${ }^{3}$ \\ ${ }^{1}$ Graduate School of Engineering, Kyoto University, Japan \\ ${ }^{2}$ School of Electrical Engineering and Automation, Wuhan University, China \\ ${ }^{3}$ Academic Center for Computing and Media Studies, Kyoto University, Japan \\ ${ }^{*}$ zhang. chi . 24e@kyoto-u.jp
}

Received: March 27, 2019; Accepted: August 8, 2019; Published: August 31, 2019

\begin{abstract}
In this paper, a system used for calculating the threshold values of extreme precipitation events and extracting these events is proposed. The threshold calculation algorithms are based on four improved percentile methods and the system selects an optimal method automatically during the calculation. In addition, the precipitation data of every year is divided into four parts according to the season. Therefore, precipitation threshold for each season is calculated and used for comparison. In experiments, the system is applied to the simulation data of precipitations from 2051 to 2111 and the variation trend of the precipitations is analyzed.
\end{abstract}

Keywords: Extreme precipitation event, Percentile method, Box-Cox transformation, Trend analysis

\section{Introduction}

The impact on global climate is deeper and deeper due to certain human activities [1]. Global climate change usually manifests itself as global warming [2], which is influencing our production and living in many aspects, such as meteorology, environment, ecological systems and so on $[3,4,5,6]$. As one of the research projects that aims at analyzing the global climate change, the SI-CAT (Social Implementation Program on Climate Change Adaptation Technology) project collaborates with local governments of Japan in developing technologies that comprehensively assess precise climate predictions and the effectiveness of its countermeasures according to their respective regional characteristics. It aims to either avoid or reduce the damage caused by climate change by reflecting the technology in future plans for urban planning, agriculture and other industries. As one of the databases used by the SI-CAT, the d4PDF (Database for Policy Decision-Making for Future Climate Change) is a public database that provides the simulation data with the main content of atmospheric 
data and meteorological data [7]. Since the d4PDF includes the future simulation data, it is possible to analyze the influence of the global climate change in the future quantitatively.

In our research, we focus on the extraction of extreme precipitation events in Japan. To achieve this goal, it is necessary to calculate the threshold value of the extreme precipitation events. Since there are some proposed methods used for calculating the threshold, we need to choose an optimal algorithm according to the data that are being used. Moreover, we also propose a new algorithm to calculate the threshold. Since the performance of these methods differs when being applied to different datasets, a system is built to select the appropriate method automatically. In addition, the data used for our research is the precipitation data in Japan, where the weather conditions have significant variation in different seasons. Therefore, the precipitation thresholds are calculated separately according to the seasons. As a result, the variation trend of different seasons can be obtained when applying the system to the analysis of the future simulation data.

The rest of this paper is organized as follows. The related work about the calculation of extreme precipitation threshold is summarized in Section 2. Section 3 describes our proposed methods in detail, including the improved percentile method based on the Box-Cox transformation and the threshold calculation system. The experimental results are presented and explained in Section 4. Finally, Section 5 presents the conclusion and discusses future work.

\section{Related Work}

The definition of extreme precipitation and the relationship between extreme precipitation and climate change was introduced in previous researches $[8,9]$. To calculate the threshold of extreme precipitation, various methods were proposed. In the early stages, a fixed extreme precipitation threshold of $50.8 \mathrm{~mm}$ per day was proposed by Karl et al. [10, 11]. Groisman et al. proposed a method to define the precipitation threshold as the absolute daily precipitation threshold of $25.4 \mathrm{~mm}$ in northern countries or $50.8 \mathrm{~mm}$ in mid-latitude countries [12]. Furthermore, threshold values for very heavy rain $(76.2 \mathrm{~mm})$ and extreme precipitation $(154.9 \mathrm{~mm})$ were used for analyzing the precipitation trend over the central United States [13].

Using a fixed value as a extreme precipitation threshold is intuitive. It is also relatively simple to calculate by using a fixed threshold value. However, in the long-term period, the climate in a region may change. If the significance of the change can not be ignored, the original extreme precipitation threshold needs to be adjusted accordingly to fit the new meteorological conditions. Because the extreme precipitation events indicate unusual events with very large amount of precipitation, if the precipitation events that exceed the threshold account for a large proportion and become the new normal, it is not appropriate to categorize them as extreme precipitation events. If extreme precipitation events are limited to a small proportion of precipitation events with the largest average daily precipitation amount during a number of years, the lower limit of the precipitation amount can be considered to be the extreme precipitation threshold. In this case, a percentile can be used to represent the proportion, which is the prototype of a percentile method [14]. In a percentile method, a cer- 
tain percentile value is selected from an ordered series of daily precipitation as the extreme precipitation threshold. The flexibility of a percentile method makes it possible to fit the circumstances of climate change. However, there is not an universal calculation method for it, which will be explained later. Another metric to quantify changes in extreme precipitation events was proposed by Kunkel et al. [15]. It is based on recurrence intervals, rather than fixed thresholds, and duration. This method mainly focus on analyzing the periodicity of extreme precipitation events. The Extreme Precipitation Index (EPI) is another index used for evaluating the regional precipitation [16]. It is the area-weighted national average frequency (per station) of extreme events for each year. This method can be applied to precipitation data in large regions, such as the United States.

For the percentile method, the settings of the percentile values are different. Karl and Knight set the percentile threshold of daily precipitation to $90 \%$ (upper 10 percentile) [17]. Pan et al. set the percentile threshold of daily precipitation to 95\% [18]. Zhai and Pan set the percentile threshold of daily precipitation to $99 \%$ [19]. The traditional percentile method assumes that the distribution of daily precipitation is uniformly distributed. However, under most circumstances, the daily precipitation data is skewed distribution. Therefore, it is necessary to adjust the traditional percentile method to improve the calculation accuracy. Luo et al. have proposed three improved percentile methods [20] based on normalization [21]. Our proposed method further improves the percentile method by adding the Box-Cox transformation to normalize the daily precipitation data. This part of work has also been presented in the Asia Simulation Conference [23].

\section{Conventional and Proposed Method}

\subsection{Improved Percentile Method}

Conventional percentile methods usually deem precipitation series to be uniformly distributed. The calculation is also based on this assumption. However, in most cases, the daily precipitation data obeys skewed distribution. The main idea of the improved percentile method is to convert the sample of precipitation data into a normal distribution. Then, the threshold corresponding to a certain percentile can be calculated via a $Z$-score. In a normal distribution, a $Z$-score is also known as a standard score and it can be placed on a normal distribution curve. Since the $Z$-score that is related to a certain percentile of precipitation can be determined, the corresponding precipitation value can also be determined by converting from the $Z$-score. The three improved percentile methods proposed by Luo et al. are summarized as Method 1, Method 2 and Method 3 as follows [20].

Method 1 In this method, the series of precipitation data is treated as a normal distribution. In this case, the $Z$-score can be calculated as

$$
\begin{gathered}
Z_{i}=\frac{6}{C_{s}}\left(\frac{C_{s}}{2} \varphi_{i}+1\right)^{\frac{1}{3}}-\frac{6}{C_{s}}+\frac{C_{s}}{6}, \\
C_{s}=\frac{\sum\left(x_{i}-\bar{x}\right)^{3}}{n s^{3}},
\end{gathered}
$$




$$
\varphi_{i}=\frac{x_{i}-\bar{x}}{s}, \quad(i=1,2, \ldots, n)
$$

where $Z_{i}$ is the $Z$-score, $C_{s}$ is the coefficient of skewness, $x_{i}$ is the daily precipitation, $\bar{x}$ is the mean value of the sample, $n$ is the sample size, $s$ is the standard deviation and $\varphi_{i}$ is the standard score. The $Z$-score related to a certain percentile can be determined by some methods such as the look-up table method. Therefore, the precipitation threshold can be calculated by the inverse transformation of the $Z$-score as

$$
x_{i}=\frac{2 s}{C_{s}}\left\{\left[\frac{C_{s}}{6}\left(Z_{i}+\frac{6}{C_{s}}-\frac{C_{s}}{6}\right)\right]^{3}-1\right\}+\bar{x} .
$$

Method 2 In this method, the series of precipitation data is transformed as

$$
y_{i}=\sqrt{x_{i}} .
$$

Then, the transformed series is used for calculating the threshold value as in Method 1. Finally, the calculated threshold value is inverse transformed by squaring and the corresponding precipitation threshold can be obtained.

Method 3 In this method, the series of precipitation data is transformed as

$$
y_{i}=\sqrt[3]{x_{i}}
$$

Then, the transformed series is used for calculating the threshold value as in Method 1. Finally, the calculated threshold value is inverse transformed by cubing and the corresponding precipitation threshold can be obtained.

In the above three methods, Method 1 directly treats the original data as normally distributed series. Method 2 and 3 use square root and cube root as the preprocessing of the original data, respectively. Here, square root and cube root are normalized transformations.

Method 4 In addition to the above three improved percentile method, we propose another improved percentile method based on the Box-Cox transformation. A Box-Cox transformation is a way to transform non-normal dependent variables into a normal shape [22]. It can be categorized as a type of power transform. The one-parameter Box-Cox transformations are defined as

$$
y_{i}^{(\lambda)}= \begin{cases}\frac{y_{i}^{\lambda}-1}{\lambda}, & \text { if } \lambda \neq 0, \\ \ln \left(y_{i}\right), & \text { if } \lambda=0,\end{cases}
$$

where $y_{i}$ is the data sample and $\lambda$ is the parameter to be determined.

The parameter $\lambda$ is estimated using the profile likelihood function. The maximum likelihood estimate of $\lambda$ is found using a special search algorithm that looks between two boundaries for the optimum value. Typically, $\lambda$ is between -5 and 5 , so the minimum is often set to -5 and the maximum is often set to 5 .

After the series of precipitation data is normalized by the Box-Cox transformation, the threshold value can be calculated as Method 1. Furthermore, the precipitation threshold can be obtained by the inverse Box-Cox transformation. 


\subsection{Precipitation Threshold Calculation System}

To evaluate the extreme precipitation threshold, it is important to evaluate its stability in different climate stages. Because the coefficient of dispersion is used for estimating the stability, it is appropriate to use it for evaluating the precipitation threshold. The coefficient of dispersion $C_{v}$ is calculated as

$$
C_{v}=\frac{s}{\bar{x}},
$$

where $s$ and $\bar{x}$ are the standard deviation and the mean value of the precipitation thresholds in different climate stages, respectively. Theoretically, a smaller coefficient of dispersion corresponds to a better calculation result of the precipitation threshold.

The moving average is used for calculating the precipitation threshold in different climate stages. For example, for a series of precipitation data during a certain period from the year $y_{1}$ to $y_{n}$, the moving average precipitation thresholds of $m$ years are calculated as follows.

1. Let the daily precipitations in the year $y_{1}, y_{2}, \ldots, y_{n}$ be $\boldsymbol{x}_{1}, \boldsymbol{x}_{2}, \ldots, \boldsymbol{x}_{n}$. The corresponding precipitation thresholds can be calculated as $t_{1}, t_{2}, \ldots, t_{n}$.

2. For $j=1$ to $j=n-m+1$, calculate the average threshold values from $t_{j}$ to $t_{j+m-1}$ as $\bar{t}_{1}, \bar{t}_{2}, \ldots, \bar{t}_{n-m+1}$.

3. The series $\overline{\boldsymbol{t}}=\left(\bar{t}_{1}, \bar{t}_{2}, \ldots, \bar{t}_{n-m+1}\right)$ is the moving average precipitation threshold values of $m$ years from the year $y_{1}$ to $y_{n}$.

As mentioned before, we need to calculate the extreme precipitation threshold in Japan. Since most regions of Japan, such as much of Honshu, Shikoku and Kyushu, belong to the temperate zone with humid subtropical climate characterized by four distinct seasons, the precipitation thresholds are calculated according to the four seasons in Japan. In our system, the precipitation data is divided into four parts by season. The moving average of precipitation thresholds for each season are calculated using the above four methods, respectively. Then, the coefficients of dispersion for the four methods are calculated and compared. The result with the minimal coefficient of dispersion is selected as the optimal precipitation threshold. Moreover, the future simulation data is included in the d4PDF. Therefore, the system can be applied to the future precipitation data and extract the extreme precipitation events in the future.

\section{Experiment and Discussion}

The data used for testing our system is the daily precipitation simulation data from the d4PDF. The period is from 2051 to 2111 and the location is Aichi Prefecture. The percentile is set to $90 \%$ (upper 10 percentile). For a specified year, the moving average values of the precipitation thresholds for one year from the previous three years to the next three years are calculated. By comparing the coefficients of dispersion, the optimal method and threshold are determined. Moreover, the indices of the optimal method for each year are recorded and shown from Fig. 1(a) to Fig. 1(d). In the figures, the horizontal axes indicate the years, 
while the vertical axes indicate the indices of methods. The indices of 1, 2, 3 and 4 represent Method 1, Method 2, Method 3 and Method 4 as mentioned above, respectively. The figures show that it is possible for each of the four methods to be chosen as the optimal method. Hence it is necessary to make a comparison to confirm which method to be used.

Furthermore, the optimal method is applied to the calculation of the moving average. The moving average values for each season's precipitation thresholds by 40 years from 2051 to 2111 are shown from Fig. 2(a) to Fig. 2(d), where the horizontal axes represent the indices of the moving average series (for example, Index 0 indicates the moving average from 2051 to 2090, Index 1 indicates the moving average from 2052 to 2091, etc.), while the vertical axes represent the precipitation thresholds. As the result, there are rising trends for the moving averages in summer and autumn, while there are downswing trends for the moving averages in spring and winter.

The experimental results show relatively clear trends of threshold moving averages in each season. Because the simulation data is based on the condition of the global warming, it is possible that the climate change causes the continuous change of the extreme precipitation thresholds. For comparison, we also calculate the total precipitations and their moving averages for 40 years in the four seasons from 2051 to 2111, respectively. As shown from Fig. 3(a) to Fig. 3(d), the total precipitations do not have clear trends. However, as shown from Fig. 4(a) to Fig. 4(d), the moving averages of total precipitations have similar trends with the moving averages of precipitation thresholds. In other words, there are rising trends for the moving averages in summer and autumn, while there are downswing trends for the moving averages in spring and winter. The obvious fluctuations in the moving average graphs correspond to the dramatic changes of the total precipitations. Further analysis is needed to confirm the relationship between the climate change and precipitation.

Moreover, the historical precipitation simulation data from the d4PDF are used to test the proposed system. The period is from 1951 to 2011 and the location is also Aichi Prefecture with the percentile being set to $90 \%$. Figure 5 shows the results with respect to the optimal calculation method in each year, where the horizontal axes indicate the years, while the vertical axes indicate the indices of methods. The indices of 1, 2, 3 and 4 represent Method 1, Method 2, Method 3 and Method 4 as mentioned above, respectively. According to Figure 1 and Figure 5, some specific methods perform well in some specific seasons, such as Method 2 in summer. It is possible that more similar patterns can also be found in the figures. At the present stage, the system is designed to select the optimal method based on the coefficients of dispersion during a certain period, as what has been expained. Futher studies with respect to the patterns are catigorized as future work. In this paper, we mainly focus on the calculation methods of precipitation thresholds. Hence, to clarify the overall performance of the methods when being applied to the historical data and the future data, the average coefficients of dispersion for Method 1, Method 2, Method 3 and the optimized system in the cases of the historical data (from 1951 to 2011) and the future data (from 2051 to 2111) are listed as Table 1. According to the table, comparing to the other methods, the proposed system has the best overall performance. 


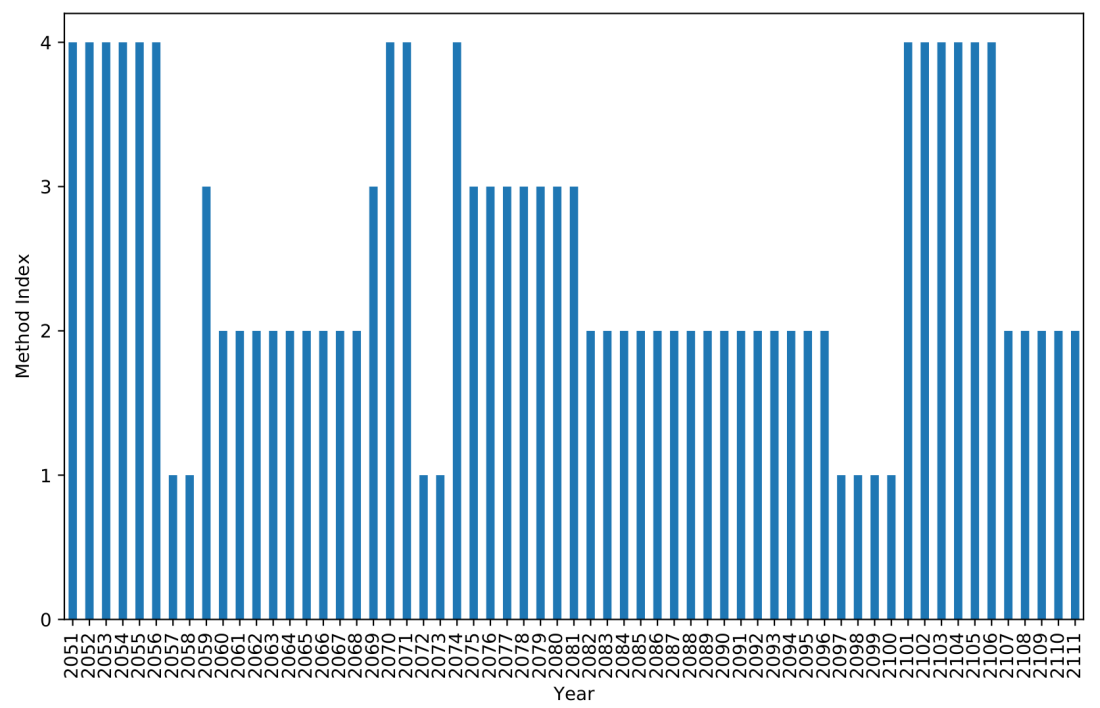

(a)

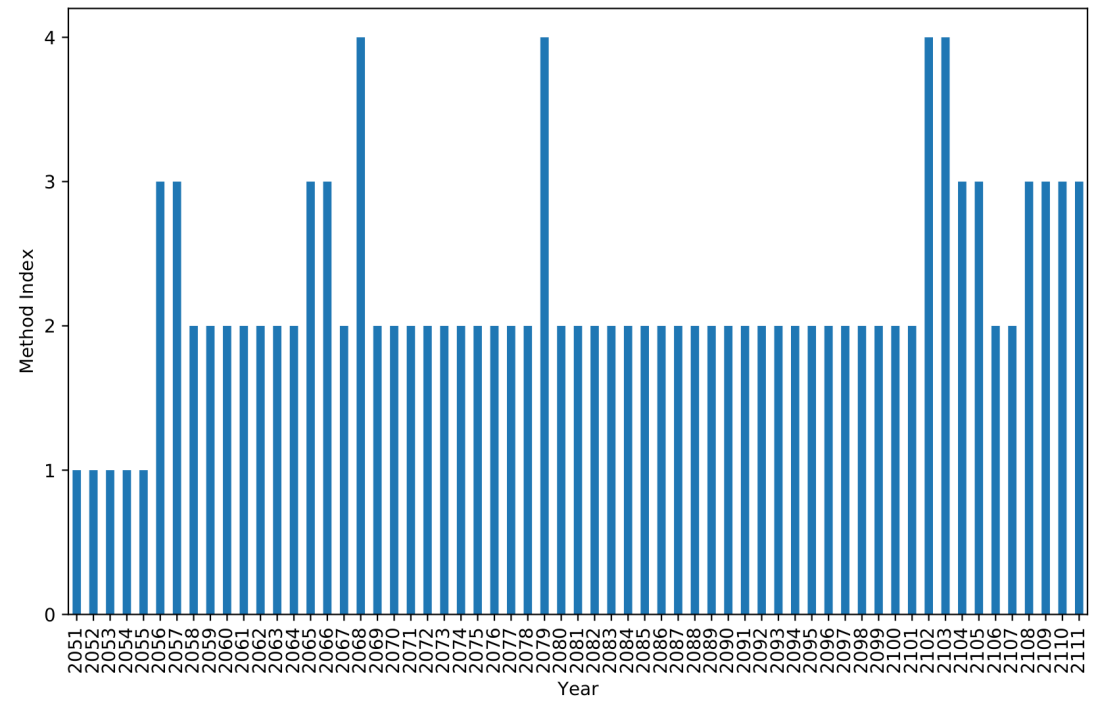

(b)

Figure 1: Optimal Threshold Calculation Methods in Different Seasons. (a) Spring. (b) Summer. 


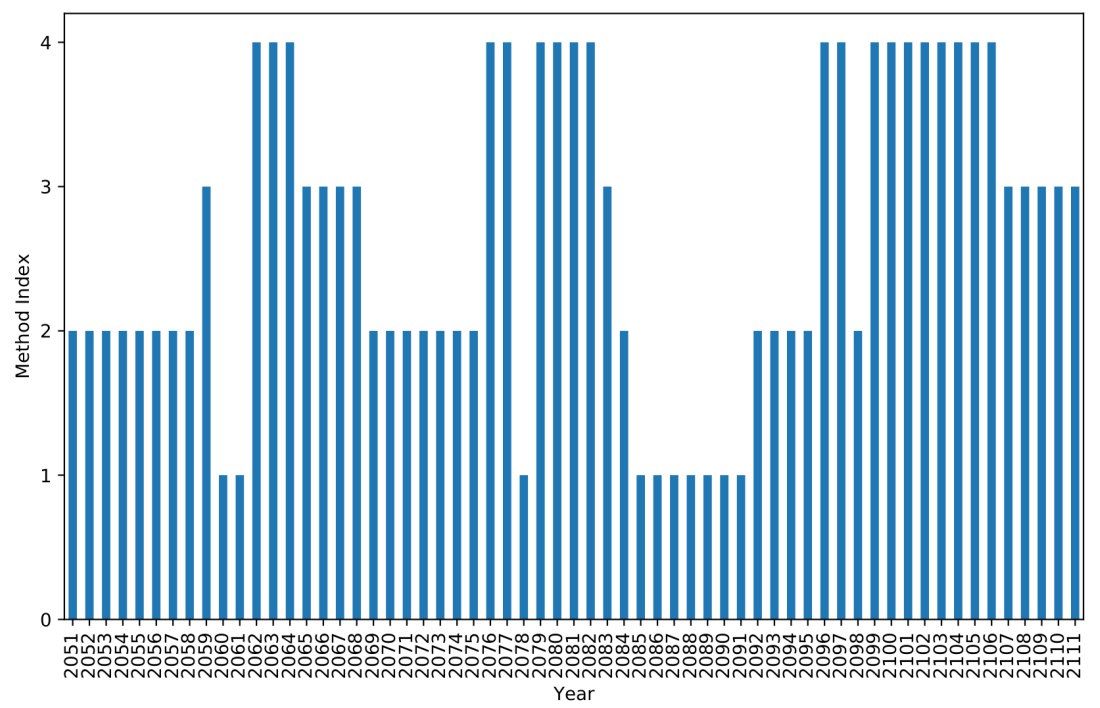

(c)

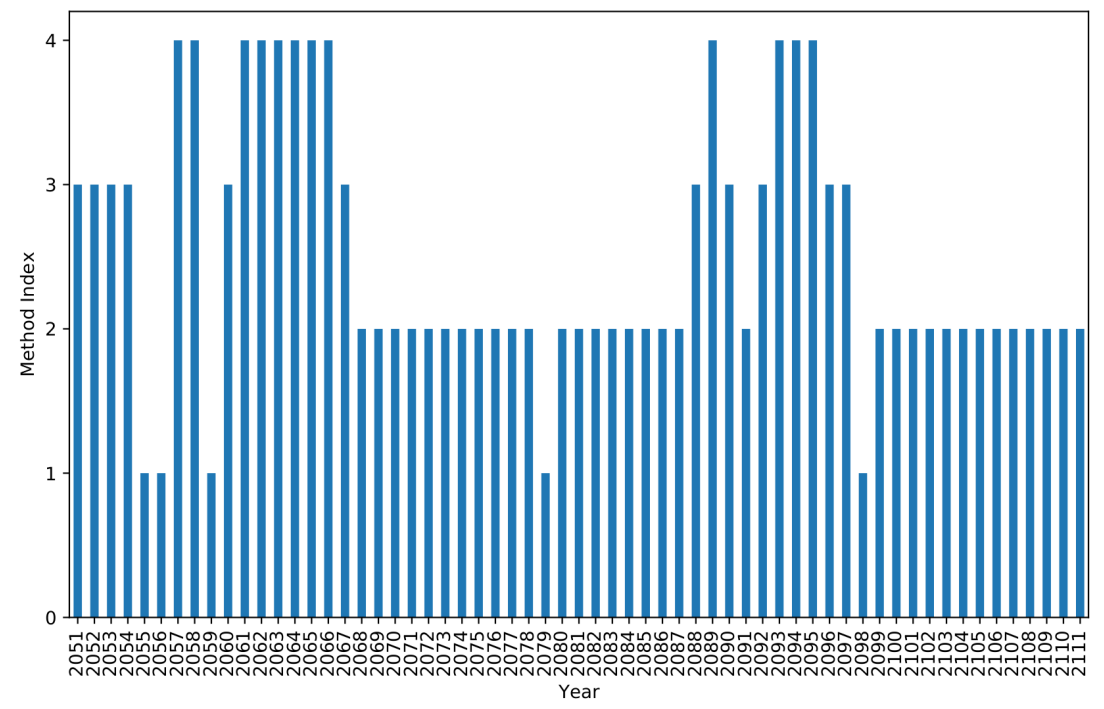

(d)

Figure 1: Optimal Threshold Calculation Methods in Different Seasons (cont.). (c) Autumn. (d) Winter. 


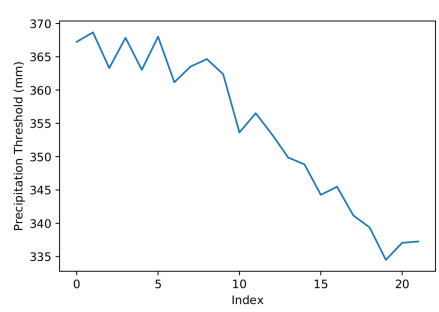

(a)

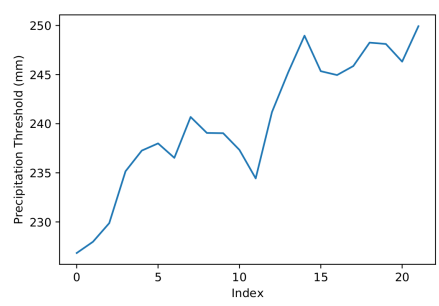

(c)

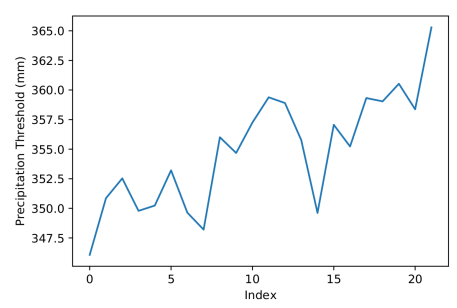

(b)

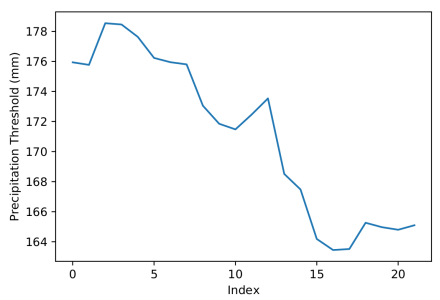

(d)

Figure 2: Moving Average Values of Extreme Precipitation Thresholds in Each Season. (a) Spring. (b) Summer. (c) Autumn. (d) Winter.

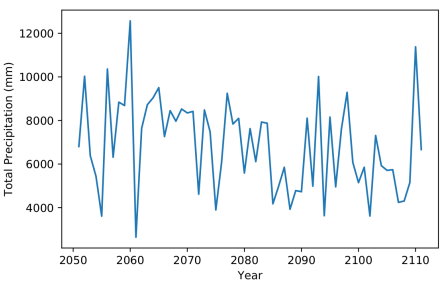

(a)

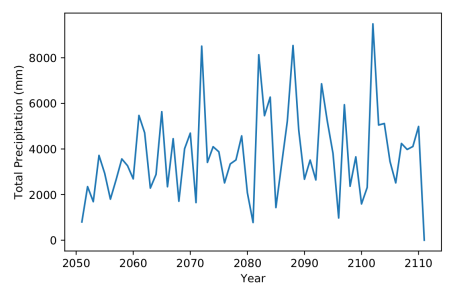

(c)

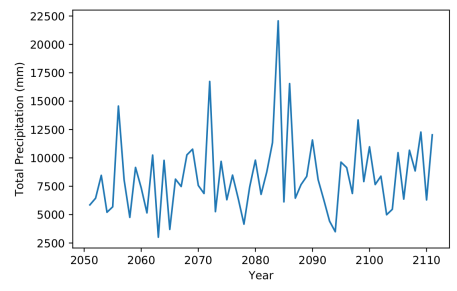

(b)

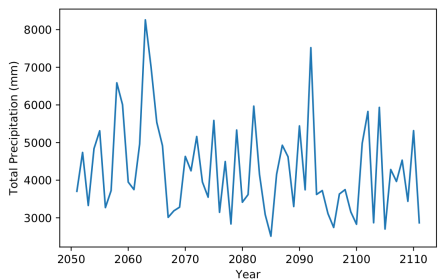

(d)

Figure 3: Total Precipitation in Each Season. (a) Spring. (b) Summer. (c) Autumn. (d) Winter. 


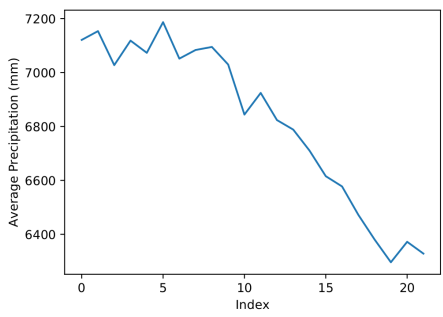

(a)

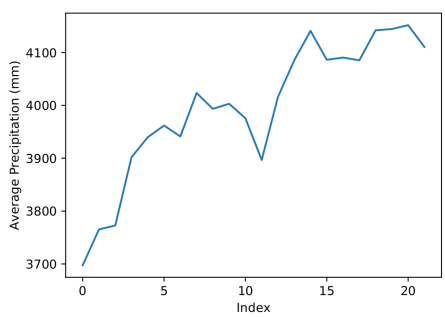

(c)

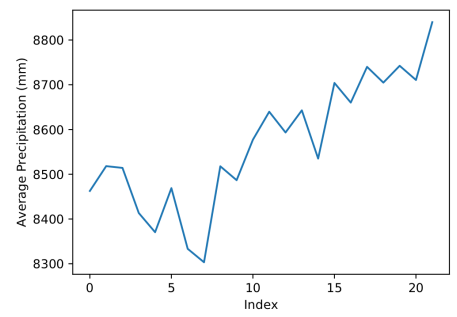

(b)

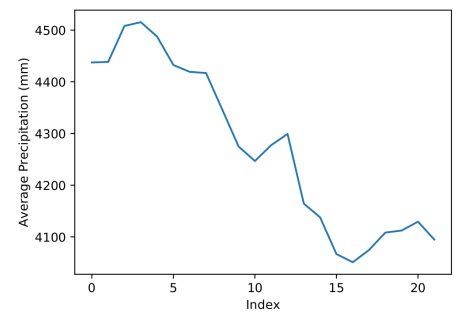

(d)

Figure 4: Moving Averages of Total Precipitations in Each Season. (a) Spring. (b) Summer. (c) Autumn. (d) Winter.

\begin{tabular}{ccccc}
\hline & Method1 & Method2 & Method3 & Optimized \\
\hline Historical & 0.3033 & 0.3004 & 0.3030 & 0.2898 \\
Future & 0.3564 & 0.3571 & 0.3663 & 0.3357 \\
\hline
\end{tabular}

Table 1: Average Cooefficients of Dispersion

\section{Conclusion and Future Work}

As complement of the existing percentile methods that are used for calculating the extreme precipitation threshold, a new method based on the Box-Cox transformation is proposed. In our system, four algorithms, including the proposed method and other three improved percentile methods, are integrated. By comparing the coefficients of dispersion, the optimal method is selected and applied to the calculation of the precipitation thresholds. Furthermore, the extreme precipitation thresholds are calculated separately corresponding to the season. In the experiment, the moving averages of extreme precipitation thresholds in each season are calculated. According to the result, rising trends are found in the moving averages of the precipitation thresholds in summer and autumn, while downswing trends are found in the moving averages of the precipitation thresholds in spring and winter. In the future, the relationship between the global climate change and the trend of extreme precipitations can be analyzed. For example, the causality between the trends of temperatures and precipitations. Moreover, extreme precipitation events also closely relate to the water levels 


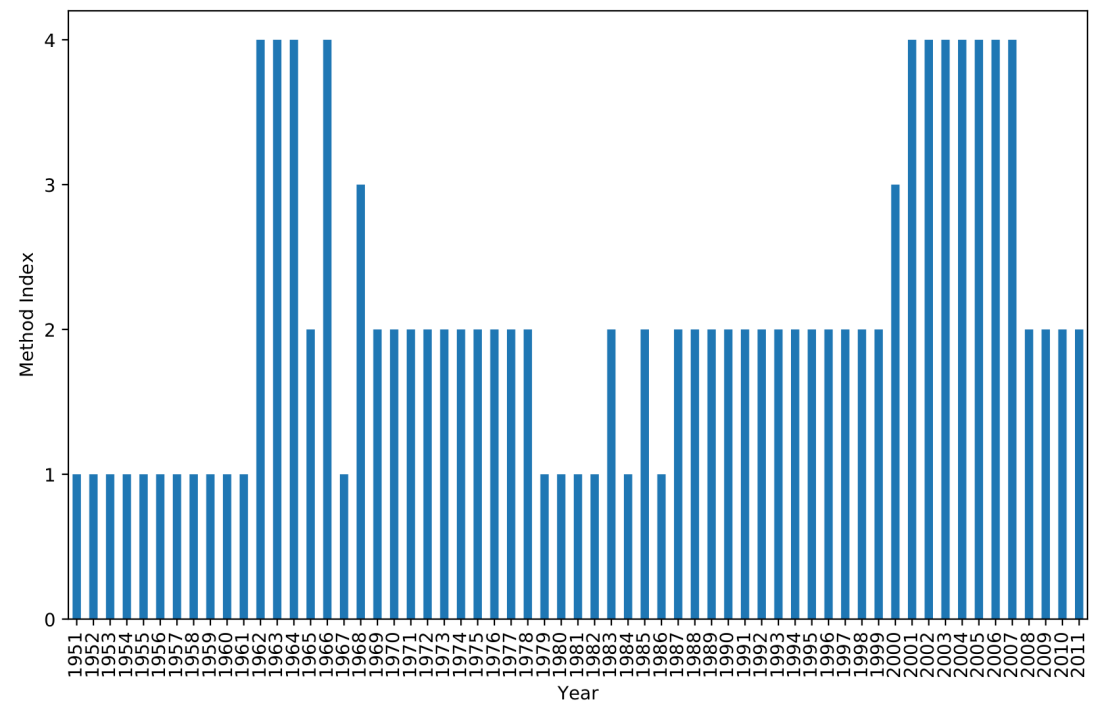

(a)

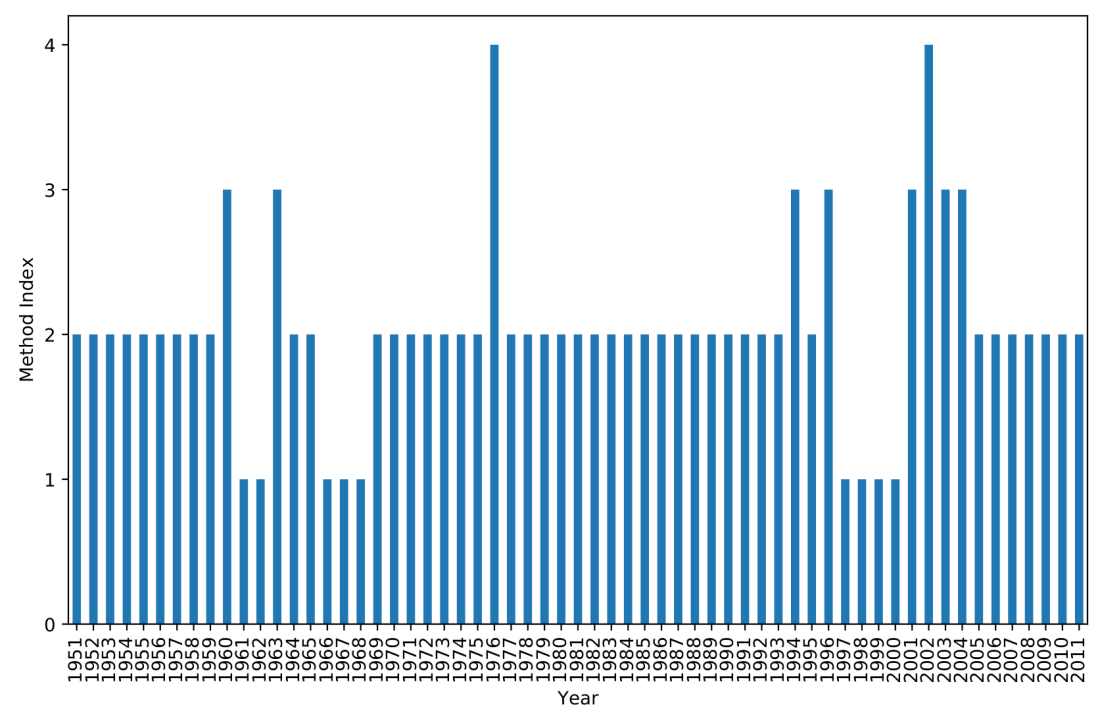

(b)

Figure 5: Optimal Threshold Calculation Methods in Different Seasons Using Historical Data. (a) Spring. (b) Summer. 


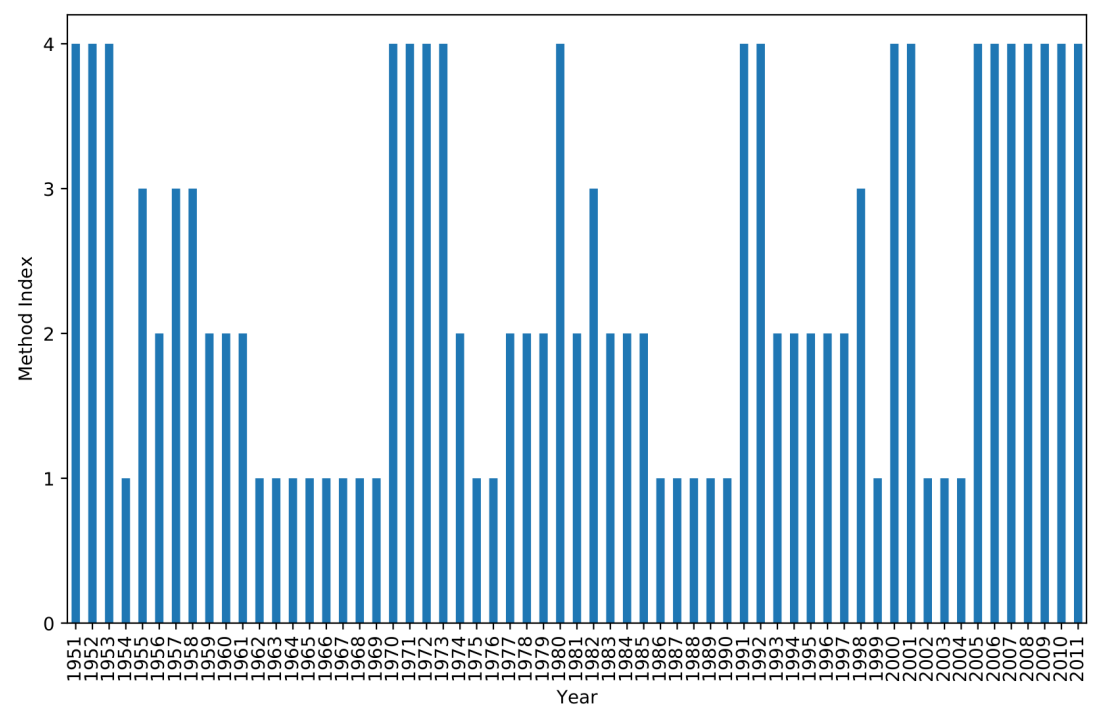

(c)

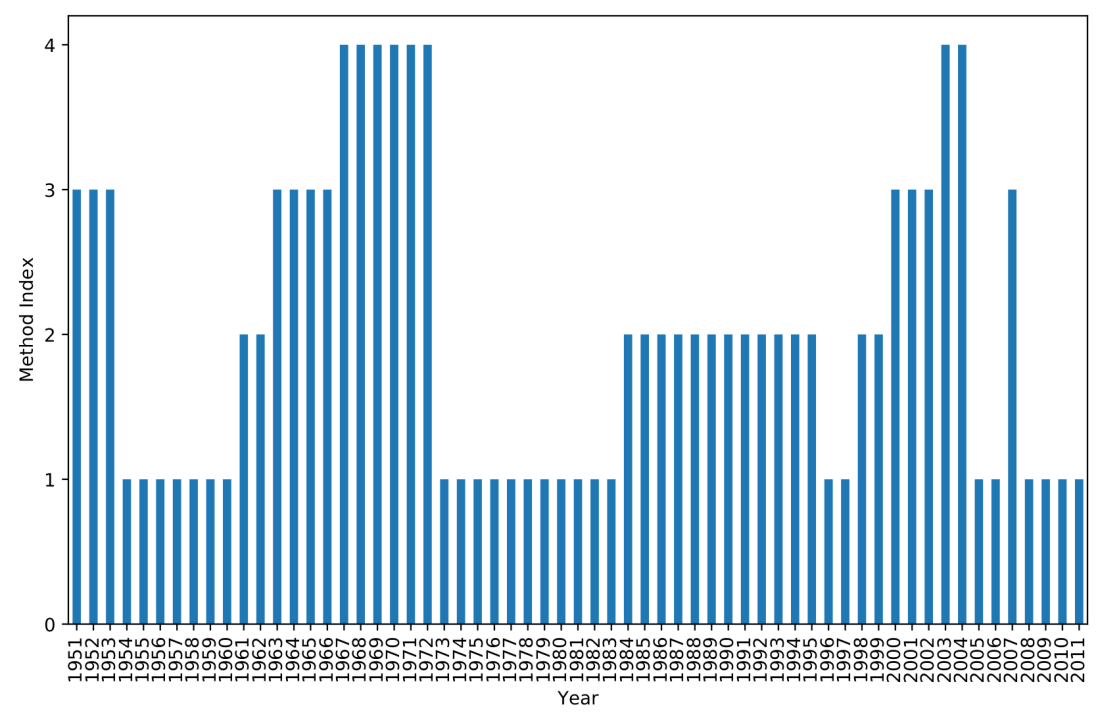

(d)

Figure 5: Optimal Threshold Calculation Methods in Different Seasons Using Historical Data (cont.). (c) Autumn. (d) Winter. 
Journal of Advanced Simulation in Science and Engineering

of rivers. It is important to predict the water level by extreme precipitation events and other parameters.

\section{Acknowledgement}

I appreciate the financial support of China Scholarship Council during my study in Kyoto University. We would also like to express our gratitude to the SI-CAT project for the support during our research.

\section{References}

[1] National Research Council: Advancing the Science of Climate Change, National Academies Press, Washington, D.C., United States, 2010.

[2] D. L. Hartmann, et al.: Observations: atmosphere and surface, Climate Change 2013 the Physical Science Basis: Working Group I Contribution to the Fifth Assessment Report of the Intergovernmental Panel on Climate Change, Cambridge University Press, Cambridge, United Kingdom, 2013.

[3] G. C. Hegerl, et al.: Understanding and attributing climate change, Climate Change 2007: The Physical Science Basis. Working Group 1 Contribution to the Fourth Assessment Report of the Intergovernmental Panell on Climate Change (IPCC) Volume Chapter 9, Geneva, Switzerland, 2007.

[4] E. M. Fischer, R. Knutti: Anthropogenic contribution to global occurrence of heavyprecipitation and high-temperature extremes, Nature Climate Change, 5:6 (2015), 560-564.

[5] C. S. Watson, et al.: Unabated global mean sea-level rise over the satellite altimeter era, Nature Climate Change, 5:6 (2015), 565-568.

[6] C. Deutsch, et al.: Climate-forced variability of ocean hypoxia, Science, 333:6040 (2011), 336-339.

[7] R. Mizuta, et al.: Over 5,000 years of ensemble future climate simulations by $60-\mathrm{km}$ global and 20-km regional atmospheric models, Bulletin of the American Meteorological Society, 98:7 (2017), 1383-1398.

[8] SK. Min, et al.: Human contribution to more-intense precipitation extremes, Nature, 470:7334 (2011), 378-381.

[9] R. S. Teegavarapu: Floods in a changing climate: extreme precipitation, Cambridge University Press, Cambridge, United Kingdom, 2012.

[10] T. R. Karl, et al.: Trends in US climate during the twentieth century, Consequences, $1: 1(1995), 3-12$. 
[11] T. R. Karl, R. W. Knight, N. Plummer: Trends in high-frequency climate variability in the twentieth century, Nature, 377:6546 (1995): 217-220.

[12] P. Y. Groisman, et al.: Changes in the probability of heavy precipitation: important indicators of climatic change, Weather and Climate Extremes, Springer, Dordrecht, 1999, 243-283.

[13] P. Y. Groisman, R. W. Knight, T. R. Karl: Changes in intense precipitation over the central United States, Journal of Hydrometeorology, 13:1 (2012), 47-66.

[14] N. Plummer, et al.: Changes in climate extremes over the Australian region and New Zealand during the twentieth century, Weather and Climate Extremes, Springer Netherlands, 1999, 183-202.

[15] K. E. Kunkel, K. Andsager, D. R. Easterling: Long-term trends in extreme precipitation events over the conterminous United States and Canada, Journal of climate, 12:8 (1999), 2515-2527.

[16] K. E. Kunkel, et al.: Temporal variations of extreme precipitation events in the United States: 1895-2000, Geophysical research letters, 30:17 (2003).

[17] T. R. Karl, R. W. Knight: Secular trends of precipitation amount, frequency, and intensity in the United States, Bulletin of the American Meteorological society, 79:2 (1998), 231-241.

[18] A. Pan, S. Fan, and H. Chen: Characteristic of extreme climate change over Jiangsu Province in the last 45 a, Scientia Meteorologica Sinica, 30:1 (2010), 87-92.

[19] P. Zhai, X. Pan: Change in extreme temperature and precipitation over northern China during the second half of the 20th century, Acta Geographica Sinica, 58:S1 (2003), $1-10$.

[20] S. Luo, S. Xiong, Y. Liang: Comparative study of calculated threshold values in regional extreme precipitation, Journal of the Meteorological Sciences, 33:5 (2013), $549-554$.

[21] J. Huang: Statistical analysis and prediction methods in meteorology, China Meteorologyical Press, Beijing, 2004.

[22] G. E. Box, D. R. Cox: An analysis of transformations, Journal of the Royal Statistical Society, Series B (Methodological) (1964), 211-252.

[23] C. Zhang, P. W. Lei, K. Koyamada: Calculation of Extreme Precipitation Threshold by Percentile Method Based on Box-Cox Transformation, in Asian Simulation Conference, Springer, Singapore, 2018, 286-295. 